\title{
Frequency Tracking with Spline Based Chirp Atoms ${ }^{\star}$
}

\author{
Matthias Wacker, Miroslav Galicki, and Herbert Witte \\ Institute for Medical Statistics, Computer Sciences and Documentation \\ Friedrich-Schiller-University Jena, GER \\ Matthias.Wacker@mti.uni-jena.de
}

\begin{abstract}
The tracking of prominent oscillations with time-varying frequencies is a common task in many signal processing applications. Therefore, efficient methods are needed to meet precision and run-time requirements. We propose two methods that extract the energy of the time frequency plane along a cubic spline trajectory. To raise efficiency, a sparse sampling method with dynamically shaped atoms is developed for the one method in contrast to standard Gabor atoms for the other. Numerical experiments using both synthetic and real-life data are carried out to show that dynamic atoms can significantly outperform classical Gabor formulations for this task.
\end{abstract}

\section{Introduction}

Frequency-dynamic oscillations are present in many situations of daily life. $\mathrm{Mu}-$ sic, wireless communication, a beating heart - endless applications deal with the task of assigning a frequency to each point in time, working against Heisenberg's uncertainty principle. Dependent on the application, requirements differ in precision, runtime, noise robustness and online applicability, leading to a variety of approaches.

Prudat and Vesin present in [1] multi-signal extensions to (FIR and IIR) notch filter based frequency tracking algorithms that minimize the filter response energy with respect to the center frequency. Similarly, Johansson and White[2] apply the notch filter concept to time variant auto regressive data modeling, leading to two types of time-variant notch filters that are applied to signals with low signal-to-noise ratios. In [3], Streit and Barrett formulate the problem of frequency line tracking as an optimal path through fixed frequency states, determined by a Hidden Markov Model which implies a high noise robustness at the cost of a coarse frequency resolution (prior chosen states). Witte et al. [4] use an active contour model approach from image processing, the Snake algorithm, to extract a time frequency line. This is the closest work to ours and will be commented on later in this paper.

In our application, we want to adapt further automatic processing steps to the time frequency course of a salient oscillation in magnetoencephalogram data. Although this oscillation is prominent, it might have discontinuations so that there

\footnotetext{
* This work was supported by DFG Wi 1166/9-1 (Gamma).
} 
might be no useful information at a point in time. Therefore, it is an important requirement that the tracking must not drift away during such disconinuations. Accordingly, the entire temporal context must be considered simultaneously.

In the following we will present an off-line approach to time frequency tracking. The general theoretic problem formulation is described in section 2, including the derivation of an efficient approach to solve it. Numerical experiments on synthetic and real-life data are performed in section 3 that lead to the final conclusion in section 4

\section{Method}

\subsection{Problem Formulation}

Formulating the goal in more mathematical terms, we look for a continuous time frequency trajectory $F \in \mathcal{C}^{0}\left[t_{0}, t_{\text {end }}\right]$ of a signal in the time range of interest. The trajectory defines a path through the complex valued time frequency plane (TFP), that can be computed by Gabor analysis [5] for a given time frequency resolution determined by $\sigma_{t}$.

$$
\operatorname{TFP}:\left\{\begin{aligned}
\mathbb{R} \times \mathbb{R} \rightarrow \mathbb{C} \\
(t, f) \mapsto \operatorname{TFP}(t, f)=\left[g\left(\tau, f, \sigma_{t}\right) * x(\tau)\right](t), \\
\quad=\int_{\tau} g\left(t-\tau, f, \sigma_{t}\right) x(\tau) d \tau
\end{aligned}\right.
$$

where

$$
g\left(t, f, \sigma_{t}\right)=\underbrace{\frac{1}{\sigma_{t} \sqrt{2 \pi}} \exp \left(\frac{-t^{2}}{2 \sigma_{t}^{2}}\right)}_{:=w_{\sigma_{t}}(t), \text { envelope }} \cdot \underbrace{\exp (i 2 \pi f t)}_{\text {compl.wave }} .
$$

The desired trajectory is defined by the path of maximum energy through the TFP

$$
\hat{F}=\underset{F \in \mathcal{C}^{0}\left[t_{0}, t_{\text {end }}\right]}{\arg \max } \int_{t_{0}}^{t_{\text {end }}}|\operatorname{TFP}(t, F(t))|^{2} \mathrm{~d} t,
$$

which implies finding the solution for an infinite dimensional optimization problem.

Beginning here, our approach differs from that of Witte et al. in 4, where the TFP is sampled and is then regarded as a discrete image, which implies a fixed frequency spacing and the computation of many points in the TFP that will remain untouched in the end. After this sampling, an active contour model is applied, which then already works on the discrete image approximation and suffers from sampling artefacts. In contrast, we regard the TFP as a function, that can be precisely evaluated at every time and frequency point. The method of energy extraction is discussed in the following.

To limit dimensionality, we reduce the search space of possible trajectories to smooth functions, which can be approximated by cubic splines [6]. Those splines automatically ensure a high degree of robustness regarding the previously mentioned discontinuations of the oscillations because the (local) change of a 
node affects the whole spline. Hence, a local overfitting can not be preferred over global information.

In order to define the spline, we choose the natural boundary condition and an equidistant spacing $\triangle_{t}$, leading to $N=\left(t_{\text {end }}-t_{0}\right) / \triangle_{t}+1$ nodes $t_{k}$.

$$
F \in\left\{\begin{array}{l|l}
s(t) & \begin{array}{c}
s(t)=a_{k}+b_{k}\left(t-t_{k}\right)+c_{k}\left(t-t_{k}\right)^{2} \\
+d_{k}\left(t-t_{k}\right)^{3}, \quad \forall t \in\left[t_{k}, t_{k+1}\right]
\end{array}
\end{array}\right\}
$$

The function values at these nodes define the complete spline and are therefore combined in an $N$-dimensional parameter vector $y$. This leads to the finite dimensional optimization problem

$$
\hat{y}=\underset{y}{\arg \max } \int_{t_{0}}^{t_{\text {end }}}\left|\operatorname{TFP}\left(t, F_{y}(t)\right)\right|^{2} \mathrm{~d} t,
$$

The dimensionality is now well under control, so we can have a closer look at the integral. The integration is numerically a very expensive and critical task, as it has to be repeated for every evaluation in the optimization. Therefore we approximate it by a sum of scalar products of Gabor atoms with the signal

$$
\hat{y}=\underset{y}{\arg \max } \sum_{v=0}^{V}\left|\int g\left(t_{v}-\tau, F_{y}\left(t_{v}\right), \sigma_{t}\right) x\left(t_{v}-\tau\right) \mathrm{d} \tau\right|^{2},
$$

where the atoms have an equidistant spacing. Using very few atoms in a subinterval of course does not sufficiently approximate the spline behavior, as one atom represents a constant frequency course. The obvious means to solve this problem is to reduce the spacing between the atoms until the spline behavior is well approximated, which implies a dense sampling of the trajectory in the TFP. The smarter way would be to adopt the shape of the atoms to the shape of the spline curve, so that a sparse sampling still generates a sufficient approximation.

As indicated in (2), the Gabor atom is nothing but a complex wave with a constant frequency, multiplied with a gaussian envelope $w(t)$. In algorithms such as matching pursuit, Gabor atoms have already been generalized to gaussian windowed linear chirps [7. Here, as we have cubic splines as target functions, the generalization is straight forward. By replacing the argument of the complex wave by the antiderivative $S(t)$ of the spline $s(t)$, we obtain:

$$
h\left(t, t_{v}, \sigma_{t}, y\right)=w_{\sigma_{t}}\left(t-t_{v}\right) \cdot \exp (i 2 \pi S(t)),
$$

where

$$
\begin{aligned}
S(t) & =\int_{t_{0}}^{t} s(\tau) d \tau \\
& =\left[\sum_{l=0}^{k-1} \frac{d_{l}}{4} \triangle_{t}^{4}+\frac{c_{l}}{3} \triangle_{t}^{3}+\frac{b_{l}}{2} \triangle_{t}^{2}+a_{l} \triangle_{t}\right]+\frac{d_{k}}{4} \delta_{t}^{4}+\frac{c_{k}}{3} \delta_{t}^{3}+\frac{b_{k}}{2} \delta_{t}^{2}+a_{k} \delta_{t}
\end{aligned}
$$

with $t \in\left[t_{k}, t_{k+1}\right]$ and $\delta_{t}=t-t_{k}$. Figure 1 shows an example of a Gabor analysis of a single Gabor atom and a single chirp atom. The coefficients $a_{k}, b_{k}, c_{k}, d_{k}$ are 

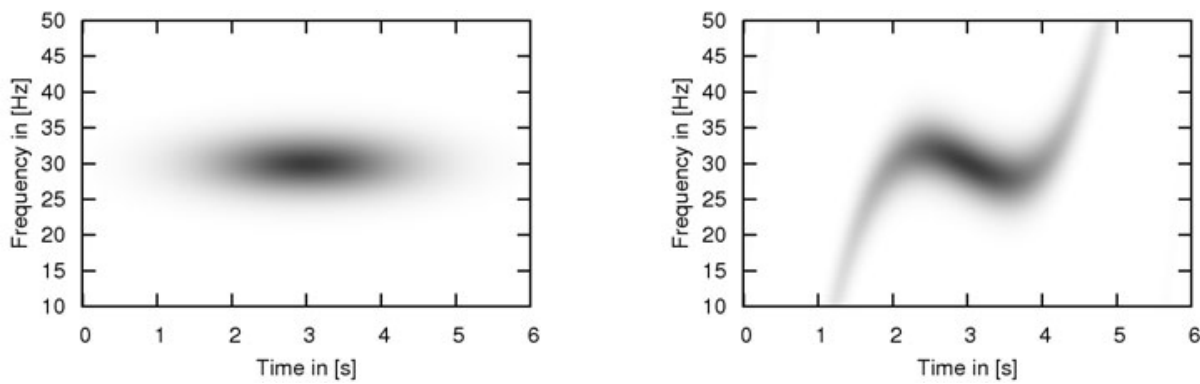

Fig. 1. Gabor analysis of a single Gabor atom (left) and a single chirp atom (right). Dark indicates high power.

uniquely defined by the spline algorithm and the spline node values $y$. So we obtain

$$
\hat{y}=\underset{y}{\arg \max } E(y)=\underset{y}{\arg \max } \sum_{v=0}^{V}\left|\int h\left(\tau, t_{v}, \sigma_{t}, y\right) x(\tau) \mathrm{d} \tau\right|^{2}
$$

as the final approach to determine the time frequency trajectory with maximum energy $E(y)$.

\subsection{Derivation of the Objective Function}

In order to implement an efficient algorithm to find the solution, we compute the derivative of the objective function with respect to the parameters. To increase readability, unnecessary indices and variables are hidden.

$$
\begin{aligned}
\frac{\mathrm{d} E(y)}{\mathrm{d} y_{j}} & =\sum \frac{\mathrm{d}}{\mathrm{d} y_{j}}\left|\int x h \mathrm{~d} \tau\right|^{2}=\sum \frac{\mathrm{d}}{\mathrm{d} y_{j}}\left[\int x h \mathrm{~d} \tau \overline{\int x h \mathrm{~d} \tau}\right] \\
& =\sum \int x \frac{\mathrm{d} h}{\mathrm{~d} y_{j}} \mathrm{~d} \tau \int x \bar{h} \mathrm{~d} \tau+\int x h \mathrm{~d} \tau \int x \frac{\mathrm{d} \bar{h}}{\mathrm{~d} y_{j}} \mathrm{~d} \tau
\end{aligned}
$$

where - indicates the complex conjugate. Next,

$$
\begin{aligned}
& \frac{\mathrm{d} h\left(t, t_{v}, \sigma_{t}, y\right)}{\mathrm{d} y_{j}}=w_{\sigma_{t}}\left(t-t_{v}\right) \exp (i 2 \pi S(t)) i 2 \pi \frac{\mathrm{d}}{\mathrm{d} y_{j}} S(t) \\
& \text { and } \frac{\mathrm{d}}{\mathrm{d} y_{j}} S(t)= \\
& =\left[\sum_{l=0}^{k-1} \frac{\mathrm{d} d_{l}}{4 \mathrm{~d} y_{j}} \triangle_{t}^{4}+\frac{\mathrm{d} c_{l}}{3 \mathrm{~d} y_{j}} \triangle_{t}^{3}+\frac{\mathrm{d} b_{l}}{2 \mathrm{~d} y_{j}} \triangle_{t}^{2}+\frac{\mathrm{d} a_{l}}{\mathrm{~d} y_{j}} \triangle_{t}\right]+\cdots \\
& \cdots+\frac{\mathrm{d} d_{k}}{4 \mathrm{~d} y_{j}} \delta_{t}^{4}+\frac{\mathrm{d} c_{k}}{3 \mathrm{~d} y_{j}} \delta_{t}^{3}+\frac{\mathrm{d} b_{k}}{2 \mathrm{~d} y_{j}} \delta_{t}^{2}+\frac{\mathrm{d} a_{k}}{\mathrm{~d} y_{j}} \delta_{t}
\end{aligned}
$$


give the derivative w.r.t. $y_{j}$ of the 'dynamic' complex atom. The derivative of $\bar{h}$ is computed in the same way. Hence, the derivation of the objective function $E(y)$ leads to the derivation of the spline coefficients w.r.t. the spline node values. To complete the computation, the following paragraph will recapitulate some facts on natural cubic splines and shows how to do the derivation.

Natural cubic splines and their derivation. For $t \in\left[t_{i}, t_{i+1}\right]$, the value of the spline $s$ is given by its $i-t h$ part

$$
\begin{aligned}
s_{i}(t) & =\frac{z_{i+1}}{6 h}\left(t-t_{i}\right)^{3}+\frac{z_{i}}{6 h}\left(t_{i+1}-t\right)^{3} \\
& +\left(\frac{y_{i+1}}{h}-\frac{h}{6} z_{i+1}\right)\left(t-t_{i}\right)+\left(\frac{y_{i}}{h}-\frac{h}{6} z_{i}\right)\left(t_{i+1}-t\right),
\end{aligned}
$$

where $h=t_{i+1}-t_{i}, z_{0}=z_{N-1}=0$ and

$$
\underbrace{\left[\begin{array}{cccc}
4 & 1 & & 0 \\
1 & 4 & & \\
& \ddots & 1 \\
0 & 1 & 4
\end{array}\right]}_{M}\left[\begin{array}{c}
z_{1} \\
\vdots \\
z_{N-2}
\end{array}\right]=\left[\begin{array}{c}
y_{2}-2 y_{1}+y_{0} \\
\vdots \\
y_{N-1}-2 y_{N-2}+y_{N-3}
\end{array}\right]
$$

Multiplying out (13) and identification with the coefficients in (4) yields

$$
\begin{aligned}
d_{i} & =\left(z_{i+1}-z_{i}\right) /(6 h) \\
c_{i} & =0.5 z_{i} \\
b_{i} & =-h z_{i} / 3-h z_{i+1} / 6+\left(y_{i+1}-y_{i}\right) / h \\
a_{i} & =y_{i} .
\end{aligned}
$$

From (14), we get

$$
\frac{\mathrm{d} z_{k}}{\mathrm{~d} y_{j}}=\left(M^{-1}\right)_{k, j-1}-2\left(M^{-1}\right)_{k, j}+\left(M^{-1}\right)_{k, j+1},
$$

where $M_{i, j}^{-1}:=0$, if $(i, j) \notin\{1, \ldots, N-2\}^{2}$ and $d z_{0} / d y_{k}=0, d z_{N-1} / d y_{k}=0$.

For the final derivation of the coefficients in (15) - (18), we obtain with the unit impulse $\delta$

$$
\begin{aligned}
\frac{\mathrm{d} d_{i}}{\mathrm{~d} y_{j}} & =\frac{1}{6 h}\left(\frac{\mathrm{d} z_{i+1}}{\mathrm{~d} y_{j}}-\frac{\mathrm{d} z_{i}}{\mathrm{~d} y_{j}}\right) \\
\frac{\mathrm{d} c_{i}}{\mathrm{~d} y_{j}} & =-0.5 \frac{\mathrm{d} z_{i}}{\mathrm{~d} y_{j}} \\
\frac{\mathrm{d} b_{i}}{\mathrm{~d} y_{j}} & =-\frac{h}{3} \frac{\mathrm{d} z_{i}}{\mathrm{~d} y_{j}}-\frac{h}{6} \frac{\mathrm{d} z_{i+1}}{\mathrm{~d} y_{j}}+\frac{1}{h}[\delta(i+1-j)-\delta(i-j)] \\
\frac{\mathrm{d} a_{i}}{\mathrm{~d} y_{j}} & =\delta(i-j) .
\end{aligned}
$$

By these computations, we completed the derivation in (12), so that now the derivation of our whole objective function is also available. 


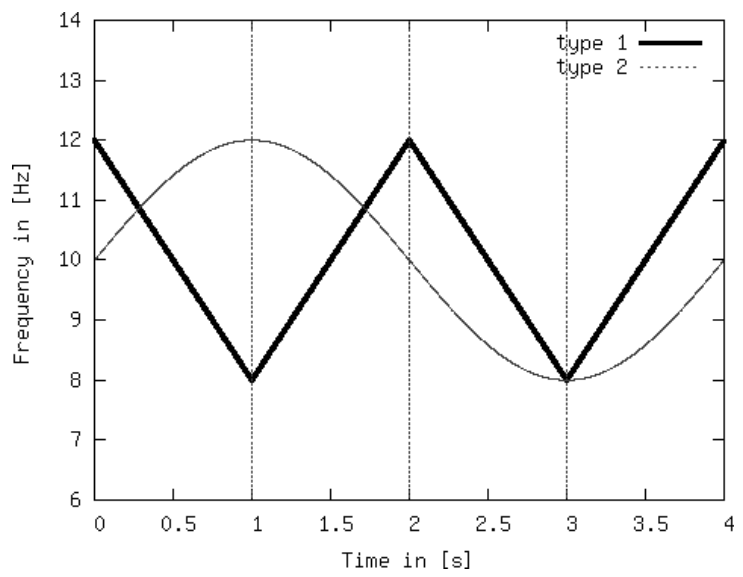

Fig. 2. Time-frequency trajectories of the benchmark signals

\subsection{Optimization}

Now that we have the derivation of our objective function, we can apply a gradient based optimization algorithm to solve (9). The class of quasi-Newton methods is of special interest, as they attempt to approximate the hessian of the objective function by an iterative update formula with the gradient information as input. Thus, these algorithms show a much more robust iteration behavior than the simple steepest ascent method, especially in the presence of "narrow valleys" in the parameter space.

We selected the so-called BFGS-algorithm (named from Broydon, Fletcher, Goldfarb, Shanno) as it is one of the most popular [8] and promising.

\section{Results}

An experiment with two synthetic signals is carried out to have a ground truth comparison of the sparse dynamic atoms with classic Gabor atoms. Afterwards, a result on real-life magneto encephalogram (MEG) data is presented.

For the simulation, we took one target chirp signal whose frequency trajectory is in $\mathcal{C}^{\infty}$ (sine function) and one from $\mathcal{C}^{0}$ (triangle function). These ground truth trajectories are visualized in Fig. 2. The vertical lines indicate the spline nodes that we allow for approximating the target signal. We compare the behavior of the dynamic atoms in contrast to the commonly known Gabor atoms. As a measure of this comparison, we computed the mean squared error between ground truth and the tracked trajectory.

In order to use "realistic" experimental conditions, the original signal types have been overlaid with noise of $0 \mathrm{~dB}$ SNR. Figure 3 shows the error values of both approaches plotted against the number of atoms per spline sub interval. The significant advantage the spline-based atoms compared to the Gabor atoms 


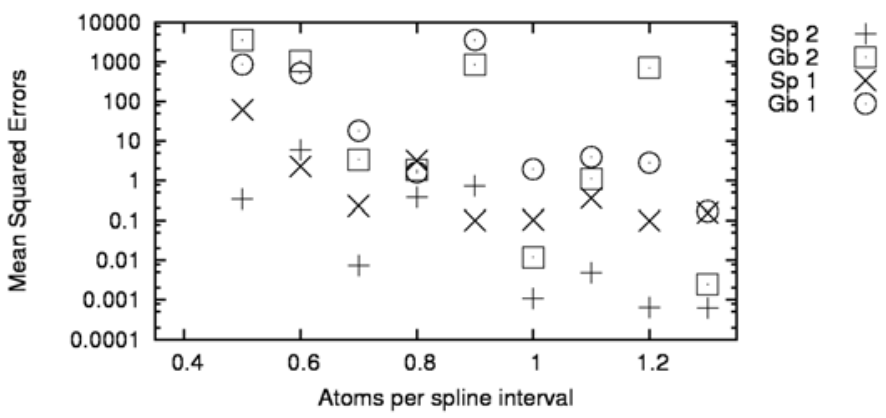

Fig. 3. Error values for spline atoms (sp) and Gabor atoms (gb) for signal type 1 and 2

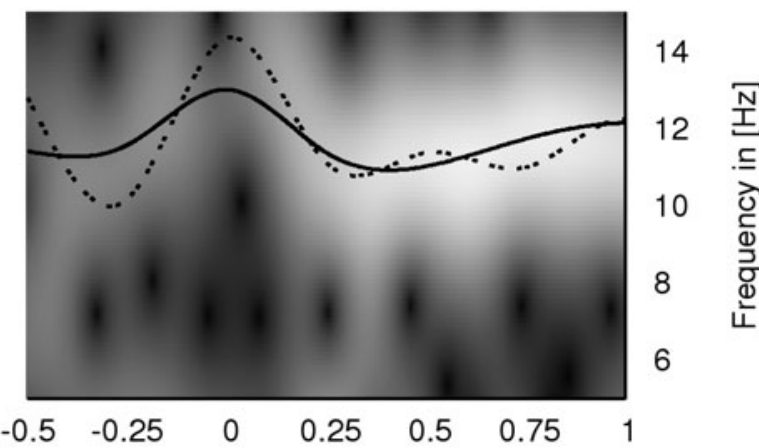

Time in [s]

Fig. 4. Time-frequency plane of a MEG-signal with the extracted trajectory as solid line for the spline atoms and as a dotted line for the standard Gabor atoms. White indicates high power.

is visible in the range where only a few atoms are used. If there are more than two atoms per subinterval, the difference between the two approaches is close to the measurement precision in our signals.

To demonstrate the practical applicability, we take MEG data from a photic driving experiment [9, where so-called alpha oscillations in the brain are manipulated by a time-limited flickering light stimulus. The dynamics of this process is of special interest to study epileptic disease. Therefore, Fig. [4 shows a Gabor analysis of the data, where white indicates high power. The onset of the stimulus is at the 0 seconds point in the presented signal. For the setup of the tracking, we chose the spline intervals to be 0.25 seconds and used one atom per interval. The result of our tracking algorithm is shown as a solid black line, the tracking with standard Gabor atoms as a dotted black line, superimposed on the Gabor analysis. It can easily be seen that the trajectory from the spline atoms outperforms that of the standard Gabor atoms. The dynamics of the oscillations are depicted clearly and more automatic data analysis is made possible at a lower computational cost. 


\section{Conclusion}

We have presented two approaches to off-line frequency tracking. The trajectory of maximum energy through the TFP is modeled by a natural cubic spline curve which enables us to reduce the search space drastically. The energy formulation is approximated by standard Gabor atoms in one case and by dynamic chirp atoms, that implicitly approximate the spline curve, in the other. The dynamic atoms allow a sparse sampling among the trajectory without losing significant precision. The derivation of these atoms with respect to the spline parameters leads to an efficient implementation using a quasi-Newton optimization scheme. Numerical experiments validate the superiority of the spline atoms over the standard Gabor atoms. Future investigations will include the design of adaptive regions of interest for higher frequency regions of MEG-data which incorporate the dynamics of the tracked alpha oscillations. The generated regions of interest will thereby be free of higher harmonics of alpha.

\section{References}

1. Prudat, Y., Vesin, J.-M.: Multi-signal extensions of adaptive frequency tracking algorithms. Signal Processing 89, 963-973 (2009)

2. Johansson, A.T., White, P.R.: Instantaneous frequency estimation at low signal-tonoise ratios using time-varying notch filters. Signal Processing 88, 1271-1288 (2008)

3. Streit, R., Barrett, R.: Frequency line tracking using hidden markov models. IEEE Transactions on Acoustics, Speech and Signal Processing 38(4), 586-598 (1990)

4. Witte, H., Putsche, P., Hemmelmann, C., Schelenz, C., Leistritz, L.: Analysis and modeling of time-variant amplitude-frequency couplings of and between oscillations of EEG bursts. Biological Cybernetics 99(2), 139-157 (2008)

5. Gabor, D.: Theory of communication. Journal of Institution of Electrical Engineers 93, 429-457 (1946)

6. Schoenberg, I.J.: On equidistant cubic spline interpolation. Bulletin of the Americal Mathematical Society 77(6), 1039-1044 (1971)

7. Kováčová, M., Kristeková, M.: New version of matching pursuit decomposition with correct representation of linear chirps. In: Proceedings of Algoritmy, pp. 33-41 (2002)

8. Nocedal, J., Wright, S.J.: Numerical Optimization. Springer, Heidelberg (2006)

9. Schwab, K., Ligges, C., Jungmann, T., Hilgenfeld, B., Haueisen, J., Witte, H.: Alpha entrainment in human electroencephalogram recordings. Neuroreport 17, 1829-1833 (2006) 